\title{
Hypericum perforatum (ethanolic extract) ameliorates simulated hypobaric hypoxia induced oxidative stress and neuronal damage in brains of Balb/c mice
}

\author{
Amardeep Gautam 1 , Rizwana Tabassum², Anju Katyal ${ }^{3}$ \\ ${ }^{1}$ Senior Research Fellow, Dr. B. R. Ambedkar Centre for Biomedical Research, University of Delhi, India, ${ }^{2}$ UGC \\ Dr. D. S. Kothari Post-Doctoral Fellow, Dr. B. R. Ambedkar Centre for Biomedical Research, University of Delhi, India, \\ ${ }^{3}$ Assistant Professor, Dr. B. R. Ambedkar Centre for Biomedical Research, University of Delhi, India
}

Background: Hypobaric hypoxia refers to lower oxygen availability at high altitudes and is the cause of high altitude illness. Drugs such as acetazolamide and dexamethasone provide symptomatic relief and are associated with undesired side effects. Plant extracts such as Hypericum perforatum, which are documented to have neuromodulatory role can be more beneficial in ameliorating high altitude illness. Aims and Objective: Progressive cognitive decline is the hallmark characteristic of hypobaric hypoxia induced neuropathology attributed to ensuing oxidative stress and subsequent hippocampal damage. We have explored the efficacy of ethanolic extracts of Hypericum perforatum in amelioration of hypobaric hypoxia induced oxidative stress and associated behavioral deficits in mice. Material and Methods: Male Balb/c mice were exposed to simulated altitude of $25,000 \mathrm{ft}$. for 7 days ( $6 \mathrm{hr}$. per day) in a specially designed chamber. Ethanolic extract of Hypericum perforatum (HPE) $(25 \mathrm{mg} / \mathrm{kg}$ of body weight) was given orally prior to Hypobaric exposure and effects were compared to Hypobaric and control groups. Results: Animals exposed to hypobaric hypoxia showed sign of cognitive deterioration at day 3 and day 7 in the Elevated Plus Maze and Passive Avoidance Step through behavioral paradigms as compare to normoxic animals. Administration of HPE was able to alleviate the amnesic effect in treatment group, indicated by reduction in transfer latencies at day $3($ IR-3 $=-0.66 \pm 0.07$ ) and day $7 \mathrm{IR}-7=-0.81 \pm 0.06$ ) in elevated plus maze task and increased passive avoidance step through latency at day $3,($ IR-3 $=3.23 \pm 0.67)$, as compared to hypoxic mice. Hypoxia group of animals suffered significant oxidative stress compared to normoxic mice as indicated by up-regulated malondialdehyde and total nitrite levels in hippocampal homogenates. The plasma lactate dehydrogenase activity was also increased following hypoxia indicating tissue damage. Co-treatment with HPE in simulated hypobaric hypoxia insult for seven days resulted in significant reduction in malondialdehyde, total nitrites and plasma LDH levels in animals. Conclusion: Hypericum perforatum extract improves cognitive performance in hypobaric hypoxia exposed mice with a concomitant reduction in oxidative stress burden suggesting its plausible use for preventing high altitude illness.

Key words: Hypobaric hypoxia, Hypericum perforatum, Cognitive deficits, Oxidative stress

\section{Access this article online}

Website:

http://nepjol.info/index.php/AJMS DOI: 10.3126/ajms.v9i2.18929 E-ISSN: 2091-0576 P-ISSN: 2467-9100

\section{INTRODUCTION}

Mountains have always fascinated human beings, making mountain tourism several billion dollar industry. However, mountains have one of the harshest and challenging environments on earth. With increase in altitude, the partial pressure of oxygen falls almost linearly, leading to hypoxic conditions. Due to lack of oxygen as terminal electron 
acceptor, the reactive oxygen species (ROS) generated at mitochondrial respiratory sites has been implicated in the pathophysiology of high altitude illness. ${ }^{1}$ Brain is most affected by this imbalance of redox state, owing to its higher oxygen demands and limited supply of antioxidants. ${ }^{2}$ Reduced availability of oxygen at high altitudes manifests as high altitude illness which includes acute mountain sickness (AMS), high altitude cerebral edema (HACE) and/or high altitude pulmonary edema (HAPE). Although chemoreceptors sense the lack in oxygen tension and the body deploys counter responses such as hyperventilation, tachycardia, and an increased blood flow to the brain etc., they are not sufficient to combat the decline in aerobic capacity. ${ }^{3}$

Cognitive dysfunction is observed in humans as well as animals subjected to hypobaric hypoxia. ${ }^{4,5}$ Hippocampus, part of the limbic system involved in learning and memory, is found to be a major structure affected by the hypobaric hypoxia induced oxidative stress leading to neuronal death and loss in cognitive functions. ${ }^{6}$ Various drugs such as acetazolamide, ibuprofen and dexamethasone providing symptomatic relief have been used to alleviate HAI. ${ }^{7}$ But pharmaceutical drugs always have their limitations and side effects. Therefore a natural approach of using herbal medicines such as plant extracts could be much more beneficial.

Hypericum perforatum, also known as St. John's Wort, is a herb used since ancient time in treatment of many ailments such as wound healing, neuralgia, fibrositis, anxiety and other psychiatric ailments. Medicinally active constituents of St. John's Wort include phloroglucinols, naphthodianthrones, flavonoids, biflavons, fluoroglucinols, xanthones, essential oils etc. ${ }^{8}$ Extracts of Hypericum perforatum (HPE) possess antioxidant properties against hydrogen peroxide induced toxicity and can be effective in treating neurodegenerative diseases. ${ }^{9}{ }^{10}$ Flavanols such as quercetin are found to be effective in neuroprotection by preventing neuronal excitotoxicity and mitochondrial dysfunction. ${ }^{11} \mathrm{H}$. perforatum was also found to inhibit monoamine oxidase- $\mathrm{B}$ and increase dopamine levels. ${ }^{12}$ It improves spatial working memory and thus cognitive performance in animals subjected to chronic restrain stress. ${ }^{13}$ Pre-treatment with standardized extracts of H.perforatum prevented the rotenone-induced oxidative damage in rats and increased the gene expression of antioxidant enzymes in these animals. ${ }^{14}$ Therefore, we designed the following study to evaluate the effect of HPE administration on hypobaric hypoxia induced neurotoxicity. Ethanolic extract of Hypericum perforatum was administered to animals orally thirty minutes before subjecting them to simulated hypobaric hypoxia (6hrs/day) corresponding to $25,000 \mathrm{ft}$ for seven days. Cognitive functions were evaluated on behavioral paradigms such as passive avoidance step through task and elevated plus maze task. Modulatory effect of HPE on oxidative stress markers such as malondialdehyde, total nitrites and lactate dehydrogenase activity were estimated. The study would help to devise prophylactic treatments to alleviate high altitude illness associated with hypobaric hypoxia exposure and broaden the scope of use of an already very popular and effective herbal medicine.

\section{Materials}

\section{Animals}

Male Balb/C mice, weighing 22-25 g, obtained from National Institute of Nutrition, Hyderabad were used for experiments at animal facility of Dr. B. R. Ambedkar Centre for Biomedical Research, University of Delhi, India. The animals had free access to food and water, maintained under 12 hour dark/light cycle, $25 \pm 2^{\circ} \mathrm{C}$ temperature and $50-60 \%$ humidity conditions. All experimental procedures were approved by the Institutional Animal Ethics Committee. Care was taken to use minimum number of animals and reduce their suffering as far as possible.

\section{MATERIALS AND METHODS}

Ethanolic extract of Hypericum perforatum (HPE) was procured from Dr. Willmar Schwabe Pharmaceuticals, Karlsruhe, Germany. Thiobarbituric acid (TBA), IGEPAL CA 630, Tris buffer, ethylene diamine tetraacetic acid (EDTA), $\mathrm{MgCl}_{2}$ and protease inhibitor cocktail (PIP8849) were purchased from Sigma-Aldrich (St. Louise, USA). Acetic acid, pyridine, ortho-phosphoric acid, sodium nitrite, sodium chloride, potassium dihydrogen phosphate, potassium chloride were purchased from Merck Ltd. (Mumbai, India), Sodium dodecyl sulphate (SDS) was purchased from Amresco-USA. $n$-Butanol was purchased from CDH laboratories (New Delhi, India). Nitrate reductase enzyme preparation and nitrate reductase cofactor preparation were purchased from Cayman Chemical Company, USA.

\section{Experimental Design}

Animals were randomly divided into four groups. Group I -Normoxia (N): served as normal controls. Group II - Hypoxia $(\mathrm{H})$ : animals were subjected to simulated hypobaric hypoxia corresponding to an altitude of 25,000 $\mathrm{ft}$, in a specially designed and calibrated hypobaric chamber (Seven Stars, India, Model No.SS7001) by gradually drawing out air via vacuum pumps over a period of 1 hour. The rate of air withdrawal was maintained corresponding to $\sim 400 \mathrm{ft}$. $/ \mathrm{min}$. and temperature was maintained at $25 \pm 2^{\circ} \mathrm{C}$. Animals were kept under these conditions for 6 hours/ day for 7 days and they had free access to food and water. Group III-Hypoxia + H. perforatum (H+HPE): animals were 
orally given $25 \mathrm{mg} / \mathrm{kg}$ body weight of $70 \%$ ethanolic extract of Hypericum perforatumin $0.3 \%$ carboxymethylcellulose as vehicle, 30 minutes before exposing to simulated hypobaric hypoxia. Group IV- Normoxia $+H$. perforatum $(\mathrm{N}+\mathrm{HPE})$ :animals were given HPE $(25 \mathrm{mg} / \mathrm{kg})$ in $0.3 \%$ CMC but not exposed to hypobaric hypoxia.

\section{Behavioral experiments}

\section{Passive avoidance step-through task (PA)}

The effect of $H$. perforatum on hypobaric hypoxia induced memory deficits was assessed by observing performance of mice on passive avoidance step-through task. This behavioral test is used to study associative memory in rodents. The apparatus consists of two compartments, a light chamber $(9 \times 9 \times 14 \mathrm{~cm})$ illuminated by a light source of $60 \mathrm{~W}$ power and a dark chamber $(14 \times 9 \times 14 \mathrm{~cm})$ separated by a sliding guillotine door. For habituation, all mice were allowed to explore both the chambers for $120 \mathrm{~s}$. On day 1 (Acquisition trial), each mouse was placed in the light chamber and allowed to explore it for $5 \mathrm{~s}$. Then the guillotine door was opened and time taken by the animal to enter into the dark chamber was recorded as step-through latency in seconds. In the dark chamber, the animal received an unavoidable electric shock $(0.2 \mathrm{~mA}, 50 \mathrm{~Hz}$ for $2 \mathrm{~s})$ via a grid on the floor of the dark compartment. On the $3^{\text {rd }}$ and $7^{\text {th }}$ day from acquisition trial, memory retention was tested by following the same procedure, except the electric shock in the dark chamber was not given (Retention trials). The time taken by the animal to enter the dark chamber was recorded as step-through latencies for day 3 and day 7 respectively. If the animal did not enter into the dark chamber within 120 seconds, the latency was recorded as $120 \mathrm{~s}$.

\section{Elevated plus maze task (EPM)}

The spatial memory function of animals in various groups was assessed using elevated plus-maze task. It exploits the tendency of rodents to go for a closed and safer place rather than an open one The elevated plus maze is a T-shaped apparatus with two open arms and two closed arms, facing each other, of dimensions $16 \mathrm{~cm} \times 5 \mathrm{~cm} \times 12 \mathrm{~cm}$ (for closed arm) having a central hub. The whole maze is raised by $25 \mathrm{~cm}$ from the floor. On day 1 (Acquisition trial), each mouse was placed at the end of the open arm, facing away from the central hub. The time taken by it to enter into the closed arm was recorded as transfer latency, in seconds. If the animal did not enter the closed arm for $120 \mathrm{~s}$, it was gently helped into the closed arm and latency was recorded as $120 \mathrm{~s}$. After entering into the closed arm, the animal was allowed to explore it for $15 \mathrm{~s}$, after which it was removed and placed back into the cage. On the $3^{\text {rd }}$ and $7^{\text {th }}$ day (retention trials), memory retention trials were done by following the same procedure as day 1 .
Inflexion ratios (IR) for passive avoidance step through and elevated plus maze tasks were calculated by using the formula:

$\mathrm{IR}=\{($ Day X latency- Day 1 latency $) /$ Day 1 latency $\}$

If $X=$ Day 3, Inflexion Ratio $=$ IR-3

If $\mathrm{X}=$ Day 7, Inflexion Ratio $=$ IR-7

\section{Biochemical experiments}

Hippocampal homogenate and plasma preparation

After behavioral studies, the animals were anesthetized using $80 \mathrm{mg} / \mathrm{kg} \mathrm{b}$ wt. of Pentothal sodium. The blood from each mouse was obtained from axillary vein and collected in centrifuge tubes containing heparin $(1 \mathrm{U} / \mathrm{mL})$. It was centrifuged at 3,000 rpm for 10 minutes to obtain plasma. After taking blood, animals were euthanized and hippocampus was dissected from the brains. Hippocampus was homogenized in homogenization buffer (containing 1\% IPEGAL CA 630, $10 \mathrm{mM}$ Tris, $150 \mathrm{mM} \mathrm{NaCl}, 1.5 \mathrm{mM}$ $\mathrm{MgCl}_{2}$ and $1 \mu \mathrm{L} / 100 \mu \mathrm{L}$ protease inhibitor cocktail). The homogenate was centrifuged at $4,000 \mathrm{~g}$ for $10 \mathrm{~min}$ at $4^{\circ}$ $\mathrm{C}$ and the supernatant was stored at $-80^{\circ} \mathrm{C}$ until further used. Protein concentration was estimated by the Bradford method at $595 \mathrm{~nm}$ (Infinite200, Tecan).

\section{Lipid peroxidation}

Exposure of animals to hypobaric hypoxia leads to increase in oxidative stress related lipid peroxidation. Quantification of malondialdehyde (MDA) is widely used as a marker for lipid peroxidation. The method described by Okhawa was used to estimate the MDA formed in the present study with slight modifications. ${ }^{15}$ Briefly, hippocampal homogenate equivalent to $100 \mathrm{mg}$ protein was mixed with $1 \mathrm{ml}$ of $20 \% \mathrm{v} / \mathrm{v}$ acetic acid, ( $\mathrm{pH} 3.5$ ), $1 \mathrm{ml} 0.67 \% \mathrm{w} / \mathrm{v}$ TBA and $0.1 \mathrm{ml} 8.1 \% \mathrm{w} / \mathrm{v}$ SDS. It was heated to $95^{\circ} \mathrm{C}$ for 60 minutes and the pink color chromogen formed was extracted with $n$-butanoland pyridine (15:1 ratio). MDA formed was estimated by recording absorbance at $532 \mathrm{~nm}$ and calculating the concentration in $\mathrm{nmol} / \mathrm{mg}$ protein by using molar extinction coefficient of MDA, viz $1.56 \times 10^{5} \mathrm{M}^{-1} \mathrm{~cm}^{-1}$.

\section{Total Nitrites}

Nitric oxide (NO) is a very reactive molecule and gets converted to more stable nitrite/nitrate forms in cell. Greiss assay has been used to estimate the amount of total nitrites in a biological sample as an indication of $\mathrm{NO}$ produced. Total nitrates in the hippocampal homogenate were reduced to nitrites by Nitrate Reductase enzyme. Nitrites were then quantified by Greiss reagent $(1 \%$ sulphanilamide, $0.1 \%$ naphthyl ethylene diamine dihydro chloridein 5\% phosphoric acid). A standard curve using various concentrations of Sodium nitrite (0.625-100 nM) was prepared and the nitrite levels in the test samples were estimated. 


\section{LDH activity}

Hypobaric hypoxia induced release of $\mathrm{LDH}$ is well documented and is a marker for neuronal cytotoxicity. ${ }^{6}$ LDH activity in blood plasma was measured using LDH-Cytotoxicity Assay Kit (BioVision, USA). LDH activity was determined by measuring the increase in the amount of formazan as per the manufacturer's specification. The water-soluble formazan dye was detected spectrophotometrically at $500 \mathrm{~nm}$.

\section{Statistical analysis}

Each value represents mean \pm SEM calculated from 5-6 animals per group. One way ANOVA followed by Tukey's posttest analysis was applied and $\mathrm{p}$ value less than 0.05 were considered significant.

\section{RESULTS}

Memory retention in mice exposed to hypobaric hypoxia and co-treated with Hypericum perforatum Passive Avoidance task

Average transfer latency in the normoxia group was significantly longer at day 3 (IR-3 = 5.12 \pm 1.47 ) and

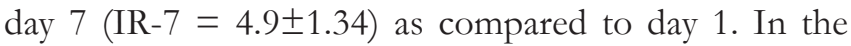
hypoxic group, there was no significant difference in day 3 (IR-3 = -0.73 $\pm 0.0 .05)$ and day 7 (IR-7 $=-0.27 \pm 0.04)$ latencies as compared to day 1 . The step-through latencies in the hypoxic compared to normoxic animals showed significant decrement on day 3 (mean difference $=67.71 \mathrm{~s}$, $\mathrm{p}<0.001$ ) as well as day 7 (mean difference $=59.01 \mathrm{~s}$, $\mathrm{p}<0.001$ ). In animals that received $H$. perforatum prior to hypoxia exposure, the latencies and inflexion ratios were comparable to normoxic controls at day 3 (IR-3 $=3.23 \pm 0.67$ ) as well as day 7 (IR-7 $=3.22 \pm 1.17$ ), these differences were not statistically significant from day 1 . However, a significant increment in inflexion ratio at day 3 in $\mathrm{H}+\mathrm{HPE}$ treated mice was observed compared to hypoxia exposed mice. In the nomoxic animals treated with H. perforatum, the inflexion ratios at day 3 and day 7 were $1.7 \pm 0.67$ and $1.83 \pm 0.67$ respectively (Figure 1 ).

\section{Elevated Plus Maze task}

Learning in mice is indicated in the elevated plus maze task by reduction in the time taken by the animal to reach the closed arm in the retrieval trials. In the normoxia group, the animals took lesser time on day 3 (IR-3 $=-0.63 \pm 0.06$ ) and on day 7 (IR-7 $=-0.26 \pm 0.09)$ as compared to day 1 . The animals exposed to hypobaric hypoxia took longer time to reach the closed arm, both at day 3 (IR-3 = 0.6 \pm 0.22 ) and at day 7 (IR-7 $=0.38 \pm 0.15)$ as compared to day 1 . The inflexion ratios at both day 3 (mean difference $=1.23$, $\mathrm{p}<0.001$ ) and day 7 (mean difference $=0.65, \mathrm{p}<0.05$ ) were significantly different among the normoxic and hypoxic animals. The mice exposed to simulated hypobaric hypoxia co-administered with $H$. perforatum for seven days, showed significant decrease in time taken to enter the

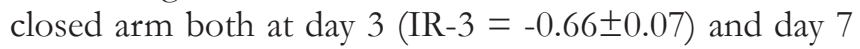
(IR-7 $=-0.81 \pm 0.06)$ as compared to non-treated hypoxic animals both on day 3 (mean difference $=1.29, \mathrm{p}<0.001$ ) and day 7 (mean difference $=1.20, \mathrm{p}<0.001)$. Animals in group IV $(\mathrm{N}+\mathrm{HPE})$ showed decreased transfer latencies as compared to day 1 , albeit statistically significant only at day 7 (IR-7 = -0.67 \pm 0.11 ) (Figure 2). The inflexion ratios in elevated plus maze task were comparable in $H$. perforatum treated hypobaric hypoxia animals and normoxic controls.

\section{Lipid peroxidation in hippocampal homogenates:}

The animals in the hypoxia group had significantly higher levels of malondialdehyde in hippocampal homogenates as compared to normoxic animals (593.6 $\pm 34.42 \mathrm{nmol} / \mathrm{mg}$ protein, $\mathrm{p}<0.001)$. Co-administration of $H$. perforatum to hypoxia exposed animals for 7 days brought down the levels of MDA in hippocampal homogenates to $194.8 \pm 29.32 \mathrm{nmol} / \mathrm{mg}$ protein $(\mathrm{p}<0.001)$. There was not much difference in the MDA levels of animals in normoxia and normoxia treatment groups (Figure 3).

\section{Total Nitrite levels in hippocampal homogenates}

There was significant increase in total nitrite levels in the hypobaric hypoxia group $(32.99 \pm 7.25 \mu \mathrm{M} / \mathrm{mg}$ protein) as compared to the normoxic animals $(14.02 \pm 2.47 \mu \mathrm{M} / \mathrm{mg}$ protein, $\mathrm{p}<0.05)$. Treatment with $H$. perforatum brought down the nitrite levels to $15.6 \pm 1.72 \mu \mathrm{M} / \mathrm{mg}$ protein, $\mathrm{p}<0.05$ (Figure 4).

\section{Lactate dehydrogenase activity}

$\mathrm{LDH}$ activity is a measure of cytotoxicity. Animals in the hypoxia group had significantly higher LDH activity as compared to normoxic animals (76.61 $\pm 23.86 \%$, $\mathrm{p}<0.05)$. Whereas three fold less plasma LDH activity was

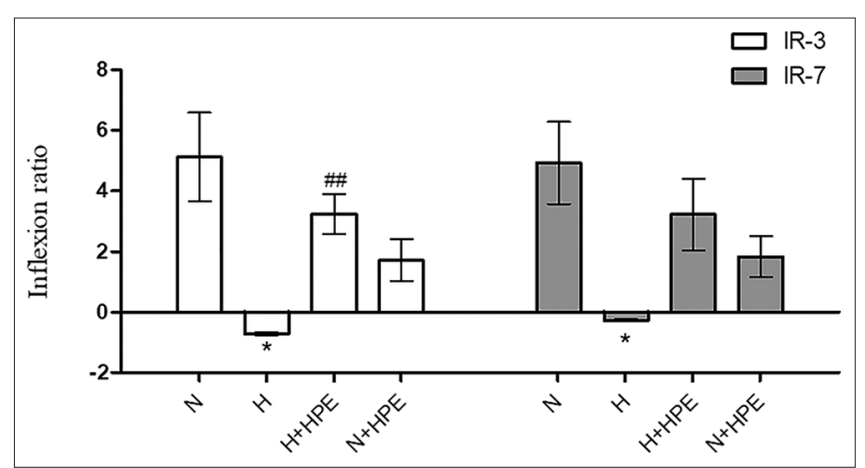

Figure 1: Modulatory effect of HPE on hypobaric hypoxia induced associative memory changes observed by passive avoidance step through task in mice. Results are shown as mean Inflexion ratios \pm SEM at subsequent retrieval trials at day 3 and day 7 . IR-3 = Inflexion ratio at day $3, \mathrm{IR}-7=$ Inflexion ratio at day 7 . One way ANOVA followed by Tukey's multiple comparision test was applied for IR-3 and IR-7 separately. For Day 3- " $\mathrm{p}<0.05 \mathrm{~N}$ vs. H; ${ }^{\#} \mathrm{p}<0.05 \mathrm{H}$ vs. H+HPE. For Day $7-{ }^{*} p<0.05 \mathrm{~N}$ vs. H. $\mathrm{N}=6$ 


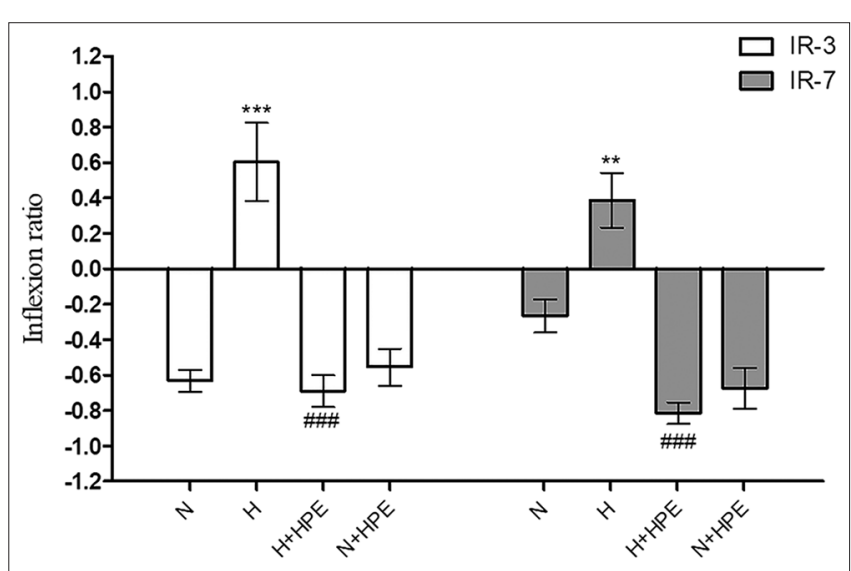

Figure 2: Effect of $H$. perforatum co-administration on elevated plus maze task performance of mice exposed to hypobaric hypoxia. Results are shown as inflexion ratios \pm SEM, IF-3 $=$ Inflexion ratio at day 3 , IF-7 $=$ Inflexion ratio at day 7.One way ANOVA followed by Tukey's Multiple Comparison Test was applied for IR-3 and IR-7 separately. For Day $3^{* * *} p<0.001 \mathrm{~N}$ vs. H; \#\#\# $p<0.001 \mathrm{H}$ vs. H+HPE. For Day $7{ }^{* *} \mathrm{p}<0.05$ $\mathrm{N}$ vs. $\mathrm{H} ;{ }^{\#} \# \mathrm{p}<0.001 \mathrm{H}$ vs. $\mathrm{H}+\mathrm{HPE}$. $\mathrm{N}=6$

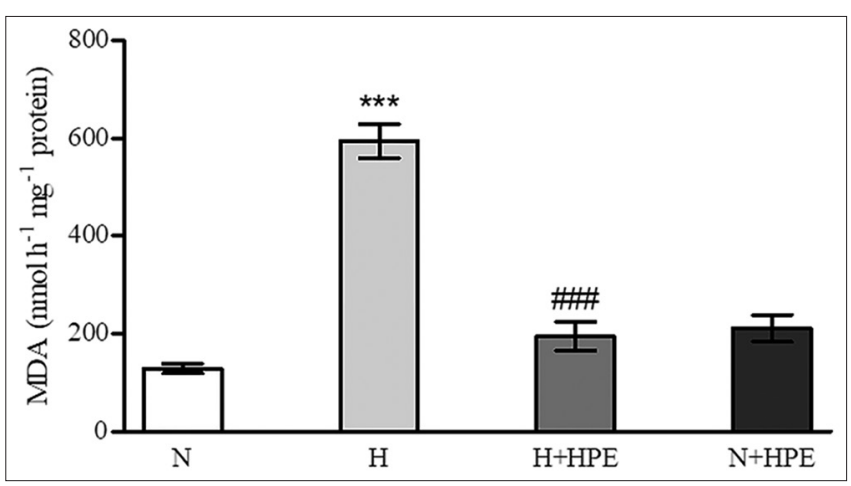

Figure 3: $H$. perforatum mediated modulation in hippocampal Malondialdehyde (MDA) levels in hypobaric hypoxia exposed mice compared to pair matched controls. Amount of MDA formed in nmol $\mathrm{mg}^{-1}$ protein by using molar extinction coefficient of MDA-TBA complex $\left(1.56 \times 10^{5} \mathrm{~cm}^{2} \mathrm{mmol}^{-1}\right.$. Results expressed as means \pm SEM. ${ }^{* \star} \mathrm{p}<0.05$ $\mathrm{N}$ vs. $\mathrm{H} ;{ }^{\# \# \#} \mathrm{p}<0.05 \mathrm{H}$ vs. $\mathrm{H}+\mathrm{HPE}$. $\mathrm{N}=6$

observed in the hypoxic animals receiving $H$. perforatum $(24.86 \pm 31.43 \%)$. Animals in group IV did not show any increased cytotoxicity (Figure 5).

\section{DISCUSSION}

In the present study, we have speculated the use of Hypericum perforatum, a popular and widely used medicinal plant, in ameliorating hypobaric Hypoxia induced oxidative stress and cognitive dysfunction in mice. As the altitude increases, the partial pressure of gases is reduced linearly. At $\sim 8000 \mathrm{~m}$, the partial pressure of oxygen is $30 \%$ of its value at sea level. ${ }^{35}$ The reactive oxygen species (ROS) generated as a result of less oxygen have been implicated in the pathophysiology of intermittent hypobaric hypoxia. Animals exposed to a regimen of intermittent hypobaric hypoxia had elevated levels of various oxidative stress indices. ${ }^{16}$ Thus, maintaining the redox balance seems imperative in preventing hypobaric hypoxia induced pathology.

Hippocampus is involved in many aspects of memory formation and any damage to this region can be attributed to memory impairment. ${ }^{17}$ Animals exposed to $\mathrm{HH}$ were in fact found to have damaged neurons in the hippocampal region. ${ }^{6}$ In the present study, the learning ability of the animals exposed to hypobaric hypoxia was decreased, as reflected by increased transfer latencies and decreased escape latencies of day 3 and day 7 in EPM and PA respectively.

EPM is used to study memory and learning in rodents effectively as there is no need to manipulate appetitive behavior or use aversive stimuli. Transfer latency in this behavioral paradigm decreases as the animal learns the spatial orientation of the maze. In the present study, the normoxic animals took significantly lesser time in reaching the closed arm in $3^{\text {rd }}$ day and $7^{\text {th }}$ day retrieval trials. In contrast, in the hypoxic group, the transfer latency was increased both at day 3 and day 7, indicating impairment in memory formation in these animals. Treatment with $H$. perforatum could ameliorate this amnesic behavior as the transfer latency was decreased at day 3 and day 7 compared to day 1 . In passive avoidance task also, the normoxic animals demonstrate intact learning mechanism as indicated by increase in the transfer latencies at day 3 and day 7. They were able to associate the aversive electric stimuli with the dark chamber and therefore were hesitant in entering it. Animals in the hypoxia group had little or no such association established as indicated by the decreased step-through latencies at day 3 and day 7 . Treatment with $H$. perforatum increased the time taken by animal to enter the dark chamber, where it had received a noxious stimulus, in retrieval trials of day 3 and day 7 , indicating memory consolidation and retrieval. Klusa et al showed that the $25 \mathrm{mg} / \mathrm{kg}$ body weight dose of ethanolic extract of $H$. perforatum, although effective in preventing depressive symptoms in chronic restrain test, did not show any significant improvements in passive avoidance task in mice administered with scopolamine.$^{18}$ However, Hypericum extracts were able to improve performances in passive avoidance task and other behavioral tasks in animal subjected to chronic restrain stress. ${ }^{19}$ Ethanolic extracts of Indian Hypericum perforatum have been shown to reverse scopolamine induced memory dysfunction in mice, albeit at much higher doses than $25 \mathrm{mg} / \mathrm{kg}$ dose used in the present study. ${ }^{19}$ This dose is equivalent to a dose of $1800 \mathrm{mg} / \mathrm{kg} \mathrm{b}$ wt. in humans, which is the maximum tolerated dose in depressed patients. ${ }^{20}$ Therefore, it is encouraging to see the nootropic effect of Hypericum perforatum extracts at $25 \mathrm{mg} / \mathrm{kg}$ b wt. which can further be extrapolated to a prophylactic dose for tolerable use in 


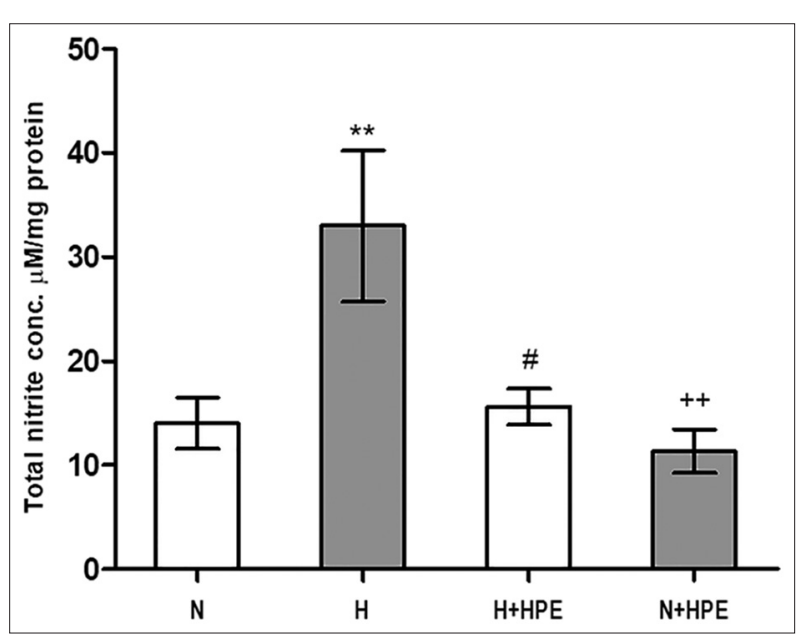

Figure 4: $H$. perforatum ameliorates the hypobaric hypoxia mediated rise in hippocampal. Concentration of total nitrite in hippocampal homogenate was calculated by using different dilutions of $\mathrm{NaNO}_{2}$ as standards $(1.5-100 \mu \mathrm{M})$. Data represented as mmole/mg protein (Mean \pm SEM). ${ }^{* *} p<0.05 \mathrm{~N}$ vs. $\mathrm{H} ;{ }^{*} \mathrm{p}<0.05 \mathrm{H}$ vs. $\mathrm{H}+\mathrm{HPE},{ }^{++} \mathrm{p}<0.05 \mathrm{H}$ vs. $\mathrm{N}+\mathrm{HPE} . \mathrm{N}=6$

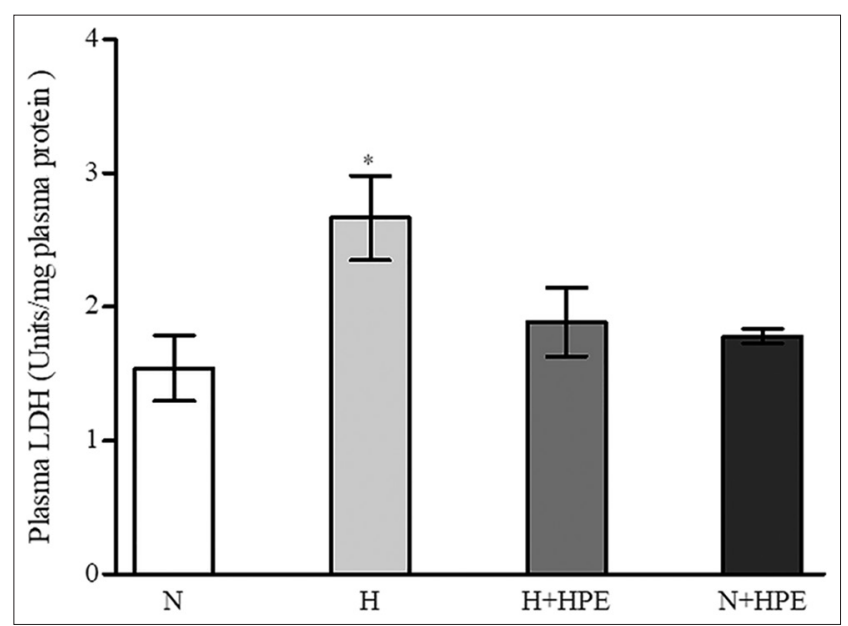

Figure 5: Lactate dehydrogenase (LDH) activity in blood plasma in $\mathrm{H}$. perforatum treated hypoxic animals compared to pair matched controls. Results expressed as units(means \pm SEM). ${ }^{*} p<0.05$ Nvs.H. $N=6$

human subjects exposed to hypobaric hypoxia.Hyperforin, one of the active constituent of Hypericum extracts has been shown to elevate the neurotransmitter levels in neurons in atypical ways by targeting the transient receptor potential channel protein 6 (TRPC6) and increasing the $\mathrm{Na}^{+}$and $\mathrm{Ca}^{2+}$ concentrations. ${ }^{21}$ The nootropic effect of Hypericum can be one of the outcomes of this altered ionic balance, especially the calcium ion.

Extracts of Hypericum rich in flavonoids demonstrate anti-oxidant properties in vitro against hydrogen peroxide induced toxicity. ${ }^{9}$ In a recent study, the hypericin deficient and flavonoid rich methanolic extracts of Hypericum connatum showed promising results in stress induced behavioral abnormalities. ${ }^{22}$ Flavonol quercetin has been shown to ameliorate the neurodegenerative effects and cognitive impairment associated with hypobaric hypoxia in rats. Daily dose of quercetin during $\mathrm{HH}$ exposure also brought down the increased levels of various oxidative stress markers. ${ }^{23} \mathrm{Zou}$ also proposed that $H$. perforatum extracts can be used in treatment of various neurodegenerative diseases where the pathology is mediated or caused by oxidative stress. ${ }^{11}$

Increased expression of nitric oxide synthase (NOS) and subsequent rise in cellular $\mathrm{NO}$ was found to be correlated with hypobaric hypoxia induced oxidative stress and DNA damage in hippocampus, and use of NOS inhibitors can be useful. ${ }^{24}$ Nitric oxide is a highly reactive molecule and in biological systems is converted to more stable nitrates. Animals exposed to simulated hypobaric hypoxia in Hypoxia group had increased total nitrite level as compared to normoxia. The co-treatment of $H$. perforatum extracts under simulated hypobaric hypoxia condition showed a significant less total nitrite levels in the hippocampal homogenates indicating animals that received $H$. perforatum extracts prior to hypoxic insult had lower NO production. In vitro studies show that flavonoids can inhibit nitric oxide (NO) production by down regulating the inducible nitric oxide synthase (iNOS) enzyme and that planar ring geometry is essential for such property. ${ }^{25}$ The flavonoid quercitin and hyperoside were found to be effective inhibitors of NOS enzymes in rat cerebrum homogenate and blood. Amongst them hyperoside was found to be selectively inhibiting the neuronal NOS. ${ }^{26}$

Lipid peroxidation is one of the markers of oxidative stress. ROS attack the lipid rich cell membranes generating peroxy free radicals. These radicals attack other lipid molecules producing more free radicals, thereby starting a chain reaction and damaging the membrane..$^{27}$ As in any other oxidative stress mediated ailment, lipid peroxidation is also observed in hypobaric hypoxia. ${ }^{16}$ In our study also, the malondialdehyde levels- marker for lipid peroxidation, were significantly higher in animals exposed to hypobaric hypoxia. Treatment with $2525 \mathrm{mg} / \mathrm{kg}$ b wt Hypericum perforatum significantly brought these levels down. Alcoholic extracts of Hypericum were found to prevent lipid peroxidation induced by chemical agents such as $\mathrm{FeSO}_{4}$ and $\mathrm{CHP}$ as well as that induced in vivo by restraint stress. ${ }^{28}$ Ethanolic extracts of Hypericum were able to reduce lipid peroxidation in vitro in hippocampal neurons..$^{29} \mathrm{H}$. perforatum extracts were also able to attenuate the lipid peroxidation in animal models of ischemia-reperfusion injury caused by bilateral occlusion of common carotid arteries. ${ }^{30}$

The key effects of ROS mediated cellular damage have been reported on plasma membrane. Release of lactate 
dehydrogenase (LDH) enzyme in the cellular milieu is one the marker for assaying membrane damage. LDH assay faithfully determines the neuronal death caused by various agents. ${ }^{31}$ In the hypoxic group, the plasma LDH activity was significantly more, indicating that there was increase cell death as a result of hypobaric hypoxia exposure. Treatment with Hypericum extracts ameliorated this cellular damage as the LDH released was significantly lowered. Hypericum extracts have been shown to reduce $\mathrm{LDH}$ release in a number of cellular stresses such as that of $\mathrm{H}_{2} \mathrm{O}_{2}$ mediated. ${ }^{20}$ ischemia/ reperfusion injury, malaria infection, tert-butyl hydroperoxide $(\mathrm{t}-\mathrm{BHP})$ etc. which is in consonance with our findings. ${ }^{32-34}$

In view of the current findings, we propose that pretreatment with Hypericum extract can prevent adverse effects of hypobaric hypoxia. A more extensive research may lead to better understanding of interaction of Hypericum perforatum with major players of oxidative stress induced at high altitude.

\section{CONCLUSIONS}

Hypericum perforatum could ameliorate the cognitive dysfunction caused by hypobaric hypoxia. The reduction in oxidative stress in Hypericum treated animals indicates that it may be acting by attenuating ROS mediated neuropathology. Hypericum seems to be a good candidate in preventing high altitude cognitive dysfunction.

This study establishes Hypericum perforatum as a potential herbal extract in treatment of high altitude illness. Hypericum already finds use in public medicine as an anti-depressant. This is first study to evaluate its nootropic effect in hypobaric hypoxia induced cognitive dysfunction.

\section{ACKNOWLEDGMENT}

The authors would like to acknowledge Indian Council for Medical Research (ICMR) for providing financial support and Scientific and Industrial Research (CSIR) for providing to Amardeep Gautam. Authors thank Dr M Udayabanu and Dr. D Kumaran for design of experiments.

\section{REFERENCES}

1. Dosek A, Ohno H, Acs Z, Taylor AW and Radak Z. High altitude and oxidative stress. Respir Physiol Neurobiol 2007; 158:128-131.

2. Shohami E, Beit-Yannai E, Horowitz M and Kohen R. Oxidative stress in closed-head injury: brain antioxidant capacity as an indicator of functional outcome. J Cereb Blood Flow Metab 1997; 17:1007-1019.

3. Bartsch P and Saltin B. General introduction to altitude adaptation and mountain sickness. Scand J Med Sci Sports 2008;18(Suppl 1):1-10.
4. Shukitt-Hale B, Kadar T, Marlowe BE, Stillman MJ, Galli RL, Levy A, et al. Morphological alterations in the hippocampus following hypobaric hypoxia. Hum Exp Toxicol 1996; 15(4):312-319.

5. Bartholomew CJ, Jensen W, Petros TV, Ferraro FR, Fire KM, Biberdorf D, et al. The effect of moderate levels of simulated altitude on sustained cognitive performance. Int J Aviat Psychol 1999; 9(4):351-359.

6. Udayabanu M, Kumaran D and Katyal A. Free chelatable zinc modulates the cholinergic function during hypobaric hypoxiainduced neuronal damage: an in vivo study. Neuroscience 2012; 202:434-445.

7. Martí-Carvajal AJ, Simancas-Racines D and Hidalgo R. Interventions for treating high altitude illness. Cochrane Database of Systematic Reviews 2012; 1. Art. No.CD009567.

8. Barnes J, Anderson LA and Phillipson JD. St. John's wort (Hypericum perforatum L): a review of its chemistry, pharmacology and clinical properties. J Pharm Pharmacol 2001; 53(5):583-600.

9. Lu YH, Du CB, Liu JW, Hong W and Wei DZ. Neuroprotective effects of Hypericum perforatum on trauma induced by hydrogen peroxide in PC12 Cells. Am J Chin Med 2004; 32(3):397-405.

10. Benedi J, Arroyo R, Romero C, Martin-Aragon S and Villar AM. Antioxidant properties and protective effects of a standardized extract of Hypericum perforatum on hydrogen peroxide-induced oxidative damage in PC12 cells. Life Sciences 2004; 75:1263-1276.

11. Silva B, Oliveira PJ, Dias A and Malva JO. Quercetin, kaempferol and biapigenin from Hypericum perforatum are neuroprotective against excitotoxic insults. Neurotox Res 2008; 13(3-4):265-279.

12. Yoshitake T, lizuka R and Yoshitake S. Hypericum perforatum L. (St John's wort) preferentially increases extracellular dopamine levels in the rat prefrontal cortex. $\mathrm{Br} \mathrm{J}$ Pharmacol 2004; 142(3):414-418.

13. Trofimiuk $\mathrm{E}$ and Braszko JJ. Alleviation by Hypericum perforatum of the stress-induced impairment of spatial working memory in rats. Naunyn Schmiedebergs Arch Pharmacol 2008; 376(6):463-471.

14. Sanchez-Reus MI, Gomezdel Rio MA, Iglesias I, Elorza M, Slowing $\mathrm{K}$ and Benedi J. Standardized Hypericum perforatum reduces oxidative stress and increases gene expression of antioxidant enzymes on rotenone-exposed rats. Neuropharmacology 2007; 52(2):606-616.

15. Ohkawa $\mathrm{H}$, Ohishi $\mathrm{N}$ and Yagi K. Assay for lipid peroxides in animal tissues by thiobarbituric acid reaction. Anal Biochem 1979; 95:351-358.

16. Udayabanua M, Kumaran D, Unnikrishnan NR, Srinivasa P, Bhagata N, Aneja R, et al. Nitric oxide associated with iNOS expression inhibits acetylcholinesterase activity and induces memory impairment during acute hypobaric hypoxia. Brain Res 2008; 138-149.

17. Huijgen $\mathrm{J}$ and Samson S. The hippocampus: A central node in a large-scale brain network for memory. Rev Neurol (Paris) 2015; 171(3):204-216.

18. Klusa V, Germane S, Noldner M and Chatterjee SS. Hypericum extract and Hyperforin: Memory-enhancing properties in rodents. Pharmacopsychiatry 2001; 34 Suppl1:S61-S69.

19. Trofimiuk E, Walesiuk A and Braszko JJ. St John's wort (Hypericum perforatum) counteracts deleterious effects of the chronic restraint stress on recall in rats. Acta Neurobiol Exp (Wars) 2006; 66(2):129-138.

20. Kumar V, Singh PN, Muruganandam AV and Bhattacharya SK. Effect of Indian Hypericum perforatum Linn on animal models of cognitive dysfunction. J Ethnopharmacol. 2000; 72(1-2):119-128.

21. Leuner K, Kazanski V, Müller M, Essin K, Henke B, Gollasch M, 
et al. Hyperforin-a key constituent of St. John's wort specifically activates TRPC6 channels. FASEB J. 2007;21(14):4101-4111.

22. Scheggi S, Marandino A, Del Monte D, De Martino L, Pelliccia T, Fusco MR, et al. The protective effect of Hypericum connatum on stress-induced escape deficit in rat is related to its flavonoid content. Pharm Biol 2016; 54(9):1782-1792.

23. Prasad J, Baitharu I, Sharma AK, Dutta R, Prasad D and Singh SB. Quercetin reverses hypobaric hypoxia-induced hippocampal neurodegeneration and improves memory function in the rat. High Alt Med Biol 2013; 14(4):383-394.

24. Maiti $P$, Singh SB and Ilavazhagan G. Nitric oxide system is involved in hypobaric hypoxia-induced oxidative stress in rat brain. Acta Hisotchemica 2010; 112(3):222-232.

25. Kim HK, Cheon BS, Kim YH, Kim SY and Kim HP. Effects of Naturally Occurring Flavonoids on Nitric Oxide Production in the Macrophage Cell Line RAW 264.7 and Their Structure-Activity Relationships. Biochem Pharmacol 1999; 58:759-765.

26. Luo L, Sun Q, Mao YY, Lu YH and Tan RX. Inhibitory effects of flavonoids from Hypericum perforatum on nitric oxide synthase. J Ethnopharmacol 2004; 93(2-3):221-225.

27. Halliwell $B$ and Chirico S. Lipid peroxidation: its mechanism, measurement, and significance. Am J Clin Nutr 1993; 57(7):15S-25S.

28. Yamini B and Pandey E. Role of alcoholic extracts of shoots of Hypericum perforatum Linn on lipid peroxidation and various species of free radicals in rats. Indian J Exp Biol 1999; 37:567-571.

29. Silva BA, Dias AC, Ferreres F, Malva JO and Oliveira CR. Neuroprotective effect of $\mathrm{H}$. perforatum extracts on beta-amyloidinduced neurotoxicity. Neurotox Res 2004; 6(2):119-130.

30. Trigunayat A. Effect of ethanolic extract of $\mathrm{H}$. perforatum on oxidative stress induced by cerebral ischemia-reperfusion in rats. Annals of Neuroscience 2009; 16(1).

31. Lobner D. Comparison of the LDH and MTT assays for quantifying cell death: validity for neuronal apoptosis? J Neurosci Methods 2000; 96:147-152.

32. Bayramoglu G, Bayramoglu A, Engur S, Senturk H, Ozturk N and Colak S. The hepatoprotective effects of Hypericum perforatum L. on hepatic ischemia/reperfusion injury in rats. Cytotechnology 2014; 66(3):443-448.

33. Zofou D, Kowa TK, Wabo HK, Ngemenya MN, Tane $P$ and Titanji VP. Hypericum lanceolatum (Hypericaceae) as a potential source of new anti-malarial agents: a bioassay-guided fractionation of the stem bark. Malar J 2011; 10:167.

34. Valentão $P$, Carvalho M, Fernandes E, Carvalho F, Andrade PB, Seabra RM, et al. Protective activity of Hypericum androsaemum infusion against tert-butyl hydroperoxide-induced oxidative damage in isolated rat hepatocytes. J Ethnopharmacol 2004; 92(1):79-84

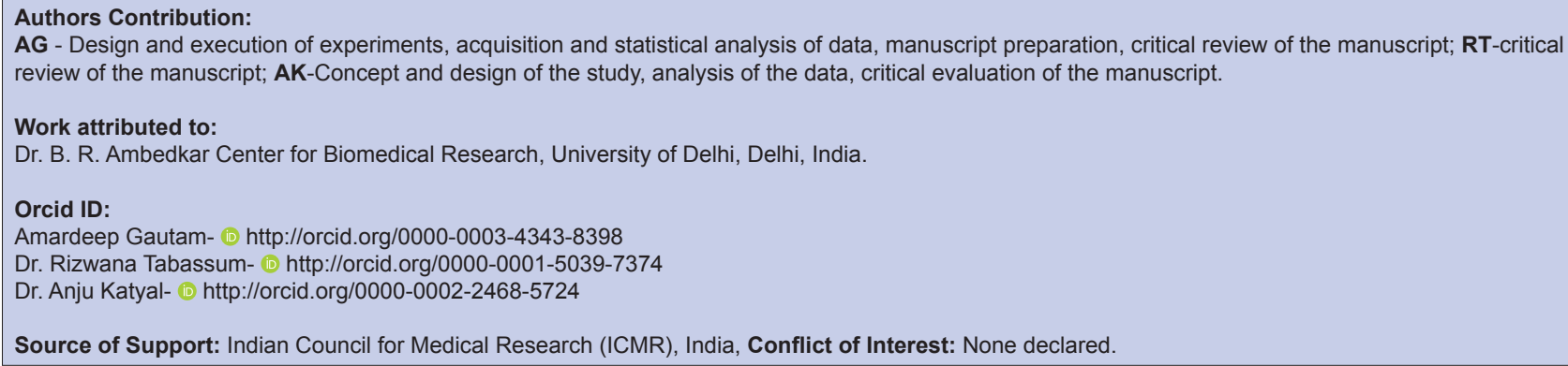

